\title{
Distinct Roles of Different Presynaptic and Postsynaptic NCAM Isoforms in Early Motoneuron-Myotube Interactions Required for Functional Synapse Formation
}

\author{
Katsusuke Hata, ${ }^{1,2 *}$ (CYuka Maeno-Hikichi, ${ }^{1 *}$ Norihiro Yumoto, ${ }^{3}{ }^{\circledR S}$ Steven J. Burden, ${ }^{3}$ and Lynn T. Landmesser ${ }^{1}$ \\ ${ }^{1}$ Department of Neurosciences, Case Western Reserve University, School of Medicine, Cleveland, Ohio 44106-4975, ${ }^{2}$ RIKEN Brain Science Institute, Wako \\ 351-0198, Japan, and ${ }^{3}$ Skirball Institute of Biomolecular Medicine, NYU Medical School, New York, New York 10016
}

The neural cell adhesion molecule (NCAM) is expressed both presynaptically and postsynaptically during neuromuscular junction formation. Genetic deletion in mice of all three isoforms $(180,140$, and $120 \mathrm{kDa})$, or just the 180 isoform, suggested that different isoforms played distinct roles in synaptic maturation. Here we characterized in mice of either sex the earliest adhesive contacts between the growth cones of motoneurons and myotubes and their subsequent maturation into functional synapses in cocultures of motoneurons and myotubes, which expressed their normal complement of NCAM isoforms, or were lacking all isoforms either presynaptically or postsynaptically. Growth cone contact with $+/+$ mouse myotubes resulted in immediate adhesive contacts and the rapid downregulation of growth cone motility. When contacting $\mathrm{NCAM}^{-1-}$ myotubes, growth cones touched and retracted/collapsed multiple times and failed to form stable contacts, even after $10 \mathrm{~h}$. Exogenous expression in myotubes of either the 180 or 140 isoform, but not the $120 \mathrm{kDa}$ isoform, rescued the rapid formation of stable contacts, the accumulation of presynaptic and postsynaptic molecules, and functional transmission. When NCAM was absent only in motoneurons, growth cones did not retract upon myotube contact, but, since their motility was not downregulated, they grew off the ends of the myotubes, failing to form synapses. The agrin receptor Lrp4 was strongly downregulated in NCAM-negative myotubes, and motoneuron growth cones did not make stable contacts with Lrp4-negative myotubes. These studies have identified novel roles for presynaptic and postsynaptic NCAM in mediating early cell-cell interactions required for synapse formation.

Key words: LRP4; motoneuron; NCAM isoforms; neuromuscular junction; synapse formation

Significance Statement

Although many molecular signals needed to form the functionally effective neuromuscular synapses required for normal movement have been described, the earliest signals that let motoneuron growth cones make stable adhesive contacts with myotubes and cease motility are not well understood. Using dynamic imaging of motoneuron-myotube cocultures, we show that NCAM is required on both the growth cone and myotube and that different NCAM isoforms mediate initial adhesion and the downregulation of growth cone motility. The agrin receptor Lrp4 was also essential for initial adhesive contacts and was downregulated on $\mathrm{NCAM}^{-1-}$ myotubes. Our identification of novel roles for NCAM and Lrp4 and possible interactions between them in transforming motile growth cones into stable contacts opens interesting new avenues for exploration.

\section{Introduction}

Synaptogenesis in both the CNS and PNS requires a series of bidirectional signaling events to organize and align the presynaptic and

Received April 14, 2017; revised Nov. 9, 2017; accepted Nov. 11, 2017.

Author contributions: K.H., Y.M.-H., and L.T.L. designed research; K.H., Y.M.-H., N.Y., S.J.B., and L.T.L. performed research; K.H., Y.M.-H., and L.T.L. analyzed data; K.H., Y.M.-H., and L.T.L. wrote the paper.

This research was supported by National Institutes of Health Grants NS-19640, NS-23678, and NS-36193 from the National Institute of Neurological Disorders and Stroke, and The Arlene H \& Curtis J Garvin endowed chair. We thank Katherine Lobur for her excellent technical assistance with the breeding and care of the mice, and Jared Cregg for help with the $\mathrm{qPCR}$ experiments.

*K.H. and Y.M.-H. contributed equally to this study.

The authors declare no competing financial interests. postsynaptic machinery required for effective transmission. The vertebrate neuromuscular junction (NMJ) has been a valuable model where several of the first organizing molecules, agrin and musclespecific kinase (MuSK), were discovered (Sanes and Lichtman, 2001; Burden, 2002). Subsequent studies identified additional organizing molecules and provided a better understanding of how they operate (Lin et al., 2001; Fox et al., 2008; Kim et al., 2008; Nishimune et al., 2008; for review, see Wu et al., 2010).

Correspondence should be addressed to Lynn T. Landmesser, Department of Neurosciences, Case Western Reserve University, 10900 Euclid Avenue, Cleveland, 0H 44106-4975. E-mail: lynn.landmesser@case.edu. DOI:10.1523/JNEUROSCI.1014-17.2017

Copyright $\odot 2018$ the authors $\quad 0270-6474 / 18 / 380498-13 \$ 15.00 / 0$ 
The neural cell adhesion molecule (NCAM), which can both mediate homophilic and heterophilic adhesion and activate intracellular signaling pathways (Maness and Schachner, 2007) is present on motoneurons and myotubes when they first establish contact and remains both presynaptically and postsynaptically at mature synapses. Although NMJs form in mice lacking all three NCAM isoforms $(180,140$, and $120 \mathrm{kDa})$, they exhibit major defects, including an inability to sustain transmission at physiological frequencies and a failure to downregulate an immature, brefeldin A-sensitive, L-type $\mathrm{Ca}^{2+}$ channel-mediated form of synaptic vesicle cycling along the axon (Polo-Parada et al., 2001; Shetty et al., 2013). While mice lacking only the 180 isoform continue to show transmission failures with repetitive stimulation, the immature form of vesicle cycling along the axon is downregulated (Polo-Parada et al., 2004), suggesting that different NCAM isoforms perform distinct roles in synaptic maturation and function.

To better define these roles, we expressed fluorescently tagged mouse NCAM isoforms in cultured chick motoneurons or myotubes and imaged them as synapses formed (Hata et al., 2007). Before myotube contact, the transmembrane 180 and 140 isoforms were localized in puncta, along the axon and growth cone, which were also sites of the immature form of synaptic vesicle cycling. Following contact, the 180 and 140 isoforms and the cycling of synaptic vesicles were rapidly and selectively targeted to the presynaptic terminal. The Glycosylphosphatidylinositol(GPI)-linked 120 isoform was more diffusely distributed and did not selectively target to the terminal after contact. This study did not, however, reveal whether NCAM was required for initial synapse formation and, if so, whether it was required presynaptically, postynaptically, or both.

To address these questions and to determine which isoforms were essential, we dynamically imaged growth cones as they contacted $\mathrm{NCAM}^{+/+}$or $\mathrm{NCAM}^{-/-}$myotubes to study the earliest anatomical adhesive interactions and the subsequent accumulation of presynaptic and postsynaptic molecules. We also characterized the consequence of the loss of presynaptic NCAM. We determined the isoforms required for effective synaptogenesis by expressing individual isoforms in NCAM-deficient cells and found that NCAM is essential for the formation of functionally effective synapses, that it is required both presynaptically and postsynaptically, and that the 140 or 180 isoforms, but not the GPI-linked 120 isoform, could mediate the stable contacts required for synapse formation. Finally, we found that the agrin receptor Lrp4 was required for motor axons to make stable contacts with myotubes and that it was downregulated on $\mathrm{NCAM}^{-1-}$ myotubes.

\section{Materials and Methods}

Mice. The $\mathrm{NCAM}^{-1-}$ mice used in these experiments were from the line described in the studies by Cremer et al. (1994) and Stork et al. (1997). The strain of origin (129P2/OlaHsd) was back-crossed into the C57BL/6 line for multiple generations and maintained locally. The wild-type (WT) mice used were from this mouse line or in some cases from pure C57BL/6 mice. In earlier experiments characterizing synaptic deficits in $\mathrm{NCAM}^{-1-}$ mice, we did not detect any differences between our $\mathrm{NCAM}^{-1-}$ line and a more extensively back-crossed $\mathrm{NCAM}^{-1-}$ line obtained from The Jackson Laboratory nor between wild-type mice from our line and wild-type pure C57BL/6 mice. We used mice of either sex in all of the experiments. All experiments were performed according to protocols approved by the Institutional Animal Care and Use Committee at Case Western Reserve University.

Preparation of wild-type and NCAM-negative mouse primary myotubes. To prepare mouse myotube cultures free of fibroblasts, muscles were dissected from eight legs of newborn mice and placed into oxygenated $\left(95 \% \mathrm{O}_{2} / 5 \% \mathrm{CO}_{2}\right)$ mouse Tyrode's solution (Rafuse et al., 2000), where connective tissue and nerve fibers were carefully removed. The remain- ing muscle was minced with a razor blade into $1 \mathrm{~mm}$ pieces and transferred to a $15 \mathrm{ml}$ tube. After brief centrifugation (1000 rpm, $5 \mathrm{~s}), 1 \mathrm{ml}$ of prewarmed papain dissociation solution (catalog \#LK003178, Worthington) was added to the pellet. After incubation in a $37^{\circ} \mathrm{C}$ incubator for 30 min, cells were washed three times with $3 \mathrm{ml}$ of growth media $[80 \%$ DMEM/F12 media (catalog \#11330-032, Life Technologies), 20\% FBS (catalog \#16000-044, Life Technologies), 5 ng/ml bFGF (catalog \#13256029, Life Technologies), and penicillin/streptomycin 1:100 (catalog \#15140-122, Life Technologies)]. The washed cells were triturated with a series of four fire-polished Pasteur pipettes with descending tip diameters (original, 1, 0.5, and $0.25 \mathrm{~mm}$ ). The cell suspension was passed through a cell strainer ( $40 \mu \mathrm{m}$; catalog \#08-771-1, Fisher Scientific) and transferred into a $50 \mathrm{ml}$ tube, and growth media were added up to $\sim 25$ $\mathrm{ml}$. Four milliliters of the cell suspension (containing $\sim 5 \times 10^{6} \mathrm{cells} / \mathrm{ml}$ ) were transferred into collagen-precoated (Purecol, catalog \#5005-B, Advanced BioMatrix) $60 \mathrm{~mm}$ dishes and placed in a tissue culture incubator at $37^{\circ} \mathrm{C}$ for $3 \mathrm{~d}$. Subsequently, the dishes were rapidly and mildly prewashed with PBS three times $(3 \mathrm{ml}, 1 \mathrm{~s} \times 3$ times). PBS $(0.75 \mathrm{ml})$ was added and then drawn back and forth very mildly into a pipette for $5 \mathrm{~min}$, or, alternatively, after incubating with PBS at room temperature $5 \mathrm{~min}$, the side of the culture dish was tapped to dislodge cells from the bottom of the dish. This procedure causes only myotubes to detach while the fibroblasts remain adherent. All glass dishes or chambers were precoated with $200 \mu \mathrm{g} / \mathrm{ml}$ poly-L-ornithine before the substrate coating to enhance the adhesion of substrate to the glass. The supernatant containing the purified myotubes is then collected, $0.25 \mathrm{ml}$ of growth media was added, and $300 \mu \mathrm{l}$ of the myotube suspension was plated onto glass bottom dishes coated with collagen or laminin (catalog \#23017-015, Life Technologies) or onto $12 \mathrm{~mm}$ precoated round glass or Thermanox plastic (catalog \#174950, Thermo Fisher Scientific) coverslips in a 24-well plate. These are placed in an incubator (usually for $1 \sim 2 \mathrm{~h}$ on laminin or overnight on collagen) until the myotubes attach to the substratum. After removal of the supernatant, $2 \mathrm{ml}$ of differentiation medium $[95 \%$ DMEM/F12 media (catalog \#11330-032, Life Technologies), 5\% heatinactivated horse serum (catalog \#26050-088, Life Technologies), $1 \mu \mathrm{M}$ hydroxyurea (catalog \#H8627, Sigma-Aldrich; Hagiwara and Ozawa, 1994)] is added. After $3 \mathrm{~d}$, these cultures contain a pure population of myotubes without contaminating fibroblasts or Schwann cells. Cell debris is washed away with Neurobasal medium (catalog \#21103-049, Life Technologies) supplemented with B27 (catalog \#17504-044, Life Technologies), without any growth factors. The myotube cultures are now ready for coculture with motoneurons.

To establish a pure myoblast population so they could be expanded for later use, the modified method by Rando and Blau (1994) was used. Briefly, hindleg muscles were collected from postnatal day 0 (P0) to P3 neonatal mice, digested with papain, washed with media, dissociated, triturated, strained as in the above procedure, and plated onto an uncoated cell culture dish first (preplate) to minimize fibroblast contamination. After $30 \mathrm{~min}$ of incubation at $37^{\circ} \mathrm{C}$ in a $5 \% \mathrm{CO}_{2}$ incubator, nonadherent cells (enriched myoblasts) were moved to a collagen-coated dish. After $3 \mathrm{~d}$ in culture and a PBS wash, the dish was tapped to collect dislodged cells, and these were preplated as described above and then transferred to a new collagen-coated dish. This purification process was repeated several times to obtain a nearly pure myoblast population, which was expanded and frozen for later use. To expand the myoblasts, HamF10 media (catalog \#N6635, Sigma-Aldrich) supplemented with $20 \% \mathrm{FBS}$ and $2.5 \mathrm{ng} / \mathrm{ml} \mathrm{FGF}$ worked better than DMEM/F12-based media because the low calcium concentration of HamF10 media $(0.3 \mathrm{~mm}$ $\mathrm{Ca}^{2+}$ ) prevented premature myotube formation and slowed fibroblast growth. Maintaining myoblasts in HamF10-based media did not change their ability to form myotubes when the media were switched to DMEM (catalog \#31600-034, Life Technologies) containing 2-5\% horse serum (up to 40 passages tested). Primary cultures of chicken myotubes were used in some experiments, and procedures for their preparation have been described in detail in the study by Hata et al. (2007).

Time-lapse imaging of growth cone myotube contacts. Cocultures of wild-type and NCAM-negative motoneurons and myotubes were prepared similarly to that used in the study by Hata et al. (2007). Briefly, cultures of purified mouse myotubes on round glass coverslips (12 mm 
diameter, described above in detail) were transferred to a $35 \mathrm{~mm}$ glassbottom microwell dish (catalog \#P35G-0-14-C, MatTek), and the medium changed to Neurobasal medium supplemented with B27. The ventrolateral portions of lumbar spinal cords of embryonic day 12.5 (E12.5) mice or stage 25 (St 25) chicken embryos were removed and explants prepared as described previously (Hata et al., 2007). Approximately 10 explants were transferred onto the myotube dish and cultured in a $37^{\circ} \mathrm{C}, 5 \% \mathrm{CO}_{2}$ incubator for $2 \mathrm{~d}$ for the motoneuron axons to extend neurites from the explant and form initial contacts with the myotubes. Explants prepared in this way consisted of $>90 \%$ motoneurons (Hata et al., 2007). Before transfer to a heated stage apparatus that maintained the culture at $37^{\circ} \mathrm{C}$, media were changed to $2 \mathrm{ml}$ of phenol red-free Neurobasal media (catalog \#12348-017, Life Technologies) supplemented with B27, and the surface was covered with light mineral oil (catalog \#ES005-C, EMD Millipore) to avoid evaporation of the media. Growth cones were selected that had extended close to individual myotubes, which were from either $\mathrm{NCAM}^{+/+}$or $\mathrm{NCAM}^{-1-}$ mice, or from $\mathrm{NCAM}^{-1-}$ mice that had been transfected with individual NCAM isoforms. Time-lapse images were acquired every minute for $10 \mathrm{~h}$ with a Nikon DIAPHOT 300 Microscope with a $40 \times$ objective lens, Retiga EXi CCD camera, and QCapture Pro Imaging Software (QImaging). Subsequent processing of images was performed with Adobe Photoshop making uniform adjustments only for brightness and contrast. For some staining experiments, myotubes and spinal cord explants were plated onto a Lab-Tek eight-well glass chamber slide (catalog \#177402, Thermo Fisher Scientific) coated first with $200 \mu \mathrm{g} / \mathrm{ml}$ poly-L-ornithine followed by $10 \mu \mathrm{g} / \mathrm{ml}$ laminin.

Immunocytochemistry. After $3 \mathrm{~d}$ in culture, cells were fixed in $3.7 \%$ formaldehyde in PBS, blocked with $0.1 \%$ Triton X-100 and $2 \%$ BSA in PBS, and stained with $\alpha$-bungarotoxin (BTX) conjugated with Alexa Fluor-488 (1:500; catalog \#B-13422, Life Technologies) as a postsynaptic marker and antibodies to SV2 (1:200; in-house preparation from Developmental Studies Hybridoma Bank) conjugated with Alexa Fluor-488 (catalog \#A20181, Life Technologies) or anti-SNAP25 (1:2000; catalog \#S-5187, Sigma-Aldrich) as presynaptic markers. To assess the targeting of the exogenously transfected NCAMs in living NCAM-knock-out (KO) myotubes or motoneurons, anti-NCAM13 monoclonal antibody, which recognizes the extracellular region of all mouse NCAM isoforms but not chick isoforms, was conjugated with Alexa Fluor 546 or 488 (Life Technologies) then was added at a final concentration of 1:200 in culture media for $15 \mathrm{~min}$, washed three times in the same media, and observed with an inverted fluorescent microscope. To show the behavior of the growth cones by immunostaining in Figure $4, E$ and $F$, spinal cord explants and myotubes are cocultured for $3 \mathrm{~d}$, then fixed, permeabilized, and stained with anti-tubulin TUJ1 antibody (1:1000; catalog \#MMS435P, Covance) and TRITC-phalloidin (40 ng/ml; catalog \#P1951, Sigma-Aldrich).

Transfection and electroporation of plasmids. Immediately after purification, NCAM-deficient myotubes on $12 \mathrm{~mm}$ round coverslips were transferred to 24 -well culture plates and $500 \mu \mathrm{l}$ of growth media without antibiotics (penicillin/streptomycin) and hydroxyurea was added. Myotubes were then transfected with plasmids of individual, fluorescently tagged isoforms of NCAM ( $1 \mu \mathrm{g} /$ well $)$ via Lipofectamine 2000 reagent (2 $\mu \mathrm{l} /$ well; catalog \#52887, Thermo Fisher Scientific) in $100 \mu$ l Opti-MEM I (catalog \#31985-070, Invitrogen) following the method recommended by the manufacturer. The three NCAM isoforms tagged with either enhanced cyan fluorescent protein (eCFP) or monomeric red fluorescent protein (mRFP1) were prepared and characterized as described previously (Hata et al., 2007). Briefly, it was shown there that when expressed in COS-7 cells, all three isoforms were expressed at comparable levels, assayed via Western blots, and that they were all capable of causing similar levels of aggregation of the COS-7 cells. This indicated that they were expressed on the cell surface and could mediate adhesion sufficient to cause cell aggregation. When expressed in chick myotubes, immunostaining with an NCAM antibody that recognizes all three mouse isoforms, but not the chick isoforms, showed that the isoforms were expressed at the cell surface at levels similar to NCAM expression on wild-type myotubes.

After $12 \mathrm{~h}$, cell debris was washed out with differentiation medium. The transfected myotubes were moved to glass-bottom dishes and used for coculture with motoneurons as described above. For transfection of motoneurons, ventral laminectomies were performed on St 25 chick or E12.5 mouse embryos in oxygenated low $\mathrm{Ca}^{2+}$ Tyrode's solution $(0.5$ $\mathrm{mm})$ and $\sim 0.5 \mu \mathrm{l}$ of NCAM plasmids $(5 \mu \mathrm{g} / \mu \mathrm{l})$ were injected via a micropipette directly into the lumbar ventral spinal cord. This was then electroporated using the protocol of $25 \mathrm{~V}, 50 \mathrm{~mA}$, five pulses, two times for chick, or $50 \mathrm{~V}, 50 \mathrm{~mA}$, five pulses, and two times for mice. The cords were removed and the ventral-lateral portions were carefully dissected and cut into $1 \mathrm{~mm}$ cubes. Approximately 7-10 explants were transferred onto pure mouse myotube cultures and kept in a $\mathrm{CO}_{2}$ incubator for the $2 \mathrm{~d}$ required for the electroporated motoneurons to express the plasmids, extend neurites, and begin making initial contacts with myotubes. For the LRP4 staining experiment, NCAM-null myoblasts were cultured on glass eight-well chamber slides coated with poly-L-ornithine and laminin on day 1 and transfected to express individual NCAM isoforms tagged with RFP, as described above, on day 2; the media were changed to cause myotube differentiation; and after $4 \mathrm{~d}$ the cultures were fixed and stained.

Imaging of growth cones contacting Lrp4-positive or Lrp4-negative mouse myotubes. LRP4 WT and KO immortalized myoblasts were established at the Burden laboratory (Kim et al., 2008). The cells were cultured and expanded in growth media (DMEM supplemented with $15 \%$ fetal bovine serum (FBS), 2\% chicken embryo extract (CEE), $1 \mathrm{~mm}$ pyruvate, $2 \mathrm{~mm}$ L-glutamine, $50 \mu \mathrm{g} / \mathrm{ml}$ gentamycin sulfate, and $2 \mathrm{ng} / \mathrm{ml}$ interferon- $\gamma$ ) on 1:10 diluted Matrigel-coated culture dishes at $33^{\circ} \mathrm{C}$ in $5 \% \mathrm{CO}_{2}$. For experiments, myoblasts were harvested and counted, adjusted to $1 \times 10^{6}$ cells $/ \mathrm{ml}$, and $50 \mu \mathrm{l}$ cells were plated on either $10 \mu \mathrm{g} / \mathrm{ml}$ laminin or 1:10 diluted. Matrigel-coated, $35 \mathrm{~mm}$ glass bottom dishes with differentiation media (DMEM supplemented with 10\% FBS, 10\% horse serum, $1 \mathrm{~mm}$ pyruvate, $2 \mathrm{~mm}$ L-glutamine, $0.5 \% \mathrm{CEE}$, and $50 \mu \mathrm{g} / \mathrm{ml}$ gentamycin sulfate) and cultured at $39^{\circ} \mathrm{C}$ in $7.5 \% \mathrm{CO}_{2}$ for $4-5 \mathrm{~d}$ to differentiate into myotubes. Coculture with WT mouse spinal cord explants was performed by culturing fully differentiated myotubes with spinal cord explants in Neurobasal Media supplemented with B27 at $37^{\circ} \mathrm{C}$ in $5 \% \mathrm{CO}_{2}$. Live cell imaging was performed on $1 \mathrm{~d}$ cocultures. Time-lapse images were taken at the minimal light intensity of the light source, at $500 \mathrm{~ms}$ exposure time with a $20 \times$ lens, using the same procedure as described above for the imaging of NCAM cocultures.

Lrp4 and $\alpha B T X$ staining of NCAM wild-type and NCAM-negative myotubes. Myoblasts were cultured in eight-well glass chambers coated with poly-L-ornithine and $10 \mu \mathrm{g} / \mathrm{ml}$ laminin with DMEM supplemented with $5 \%$ horse serum at $37^{\circ} \mathrm{C}$ and $5 \% \mathrm{CO}_{2}$. After $4 \mathrm{~d}$ for differentiation into myotubes, cells were fixed for 15 min with $3.7 \%$ formaldehyde in PBS buffer with $0.1 \%$ Triton X-100 at room temperature. Cells were washed with PBS three times and blocked with $2 \%$ BSA and $0.1 \%$ Triton X-100 in PBS (blocking buffer) at room temperature for $30 \mathrm{~min}$. The LRP4 antiserum against the extracellular N-terminal region of Lrp4 (raised in the Burden laboratory) was diluted to 1:1000 in blocking buffer and incubated with cells at $4^{\circ} \mathrm{C}$, overnight. Following washout of the primary antibody with PBS three times, a mixture of 1:200 dilution of Alexa Fluor 488-conjugated goat anti-rabbit antibody (catalog \#A-11034, Thermo Fisher Scientific) and 1:500 dilution of Alexa Fluor 594-conjugated $\alpha$ BTX (catalog \#B13423, Thermo Fisher Scientific) were applied to cells at room temperature for $1 \mathrm{~h}$. After three washes with PBS, cells were mounted in mounting media. Images of Lrp4 and AChRs on NCAMpositive and NCAM-negative myotubes were acquired with an Olympus BX51BI Fluorescence Microscope, and overall fluorescence intensity was quantified by MetaMorph (Molecular Devices) as regions of interest (ROIs) that outlined each myotube. The images used for analysis all had the same exposure time.

We noticed some nonspecific background staining with the anti LRP4 when we tested the antiserum on LRP4-null myotubes and therefore prepared an adsorbed antiserum to Lrp4 to reduce the nonspecific binding. LRP-null myotubes were cultured and fixed with 3.7\% formaldehyde in PBS, permeabilized with $0.1 \%$ Triton X-100 in PBS and blocked with $2 \%$ BSA in $0.1 \%$ Triton X-100 PBS. LRP4 antiserum was applied to the cells and kept at $4^{\circ} \mathrm{C}$ overnight. The antibody solution was collected and centrifuged to remove cell debris. The adsorbed LRP4 antiserum, which was used at 1:200 dilution, was tested on LRP4 wild-type and Lrp4-null myotubes, where we observed a reduction in background 
staining. We then used the adsorbed antisera on NCAM wild-type and NCAM-null myotubes as in Fig. 7. The pattern of staining with the LRP4 antibody before and after adsorption was not changed. The live cell staining of LRP4 on NCAM and NCAM-null myotubes was performed by incubating cells with the adsorbed LRP4 antibody (1:200) in $15 \mathrm{~mm}$ HEPES-buffered culture media, on ice for $1 \mathrm{~h}$. Following washing three times with PBS, fixation, permeabilization, blocking, and incubation with secondary antibody was performed in the same manner as the description above.

Quantitative PCR to assess Lrp4 message in NCAM wild-type and NCAM-deficient myotubes. NCAM-deficient or wild-type myoblasts were plated on either collagen-coated $35 \mathrm{~mm}$ dishes or six-well plates. Growth media were changed to differentiation media the following day, and myoblasts were allowed to differentiate into myotubes for $3 \mathrm{~d}$. mRNA was isolated from each dish or wells in plate individually using Direct-zol RNA MiniPrep (catalog \#R2050, Zymo Research) according to the manufacturer protocol. The same amount of mRNA was reverse transcribed to cDNA (catalog \#4368814, Applied Bosystems), and quantitative PCR (qPCR) was performed using iTaq Universal SYBR Green One Step Kit (catalog \#172-5150, Bio-Rad) via a Bio-Rad iCycler. The two isoforms of LRP4, LRP4-1 (exons1-12) and LRP4-4 (exons 1-38), were amplified using the following primer pairs: LRP4-1: 5' aaagctgcc cttcagcagta3' (forward) and 5' acacatgaattccccagagc3' (reverse); LRP4-4: 5'ctgcggtgtgaataatggtg3' (forward) and 5'gcactgggctcttctcattc3' (reverse). GAPDH was used as a control, and its primer pair was $5^{\prime}$ aaggtcatcccagagct gaa3' (forward) and 5' ctgcttcaccaccttcttga3' (reverse). Both relative expression and $\log 2$ ratio were calculated.

Exogenous expression of LRP4 on NCAM-negative myotubes. Fulllength LRP4 with mCherry-tagged plasmid (pCDNA3.1-Flag-LRP4mCherry; Kim et al., 2008) was transfected to NCAM-negative myoblasts according to the above transfection and electroporation method section. Transfected myoblasts were allowed to differentiate for $3 \mathrm{~d}$, then live cell staining was performed to detect the surface-expressed LRP4 as described above.

Data presentation and analysis. All data are presented as the mean \pm SE. The statistical significance was calculated by one-way ANOVA unless noted otherwise and is presented as ${ }^{* *} p<0.01$ or ${ }^{*} p<0.05$. Actual $p$ values are in the figure legends. $N$ refers to the number of cells or events counted from at least three separate experiments, unless noted otherwise

\section{Results}

\section{Characterization of growth cone behavior via dynamic imaging upon contact with myotubes}

We detected three distinct types of motoneuron growth cone behavior when contacting $\mathrm{NCAM}^{+/+}, \mathrm{NCAM}^{-/-}$, or $\mathrm{NCAM}^{-/-}$myotubes expressing the 180,140 , or 120 isoform, as follows: (1) Stable contacts: all growth cones contacting $\mathrm{NCAM}^{+/+}$myotubes or $\mathrm{NCAM}^{-/-}$myotubes exogenously expressing the 180 or 140 isoform formed stable contacts. While there was some variation in the timing of events, all progressed through the following sequence of events. Upon myotube contact, the growth cone maintained contact with the myotube at the site of initial contact and mediated adhesion sufficient to maintain contact even though myotubes exhibited considerable movement. Within 10-96 min of initial contact, a boutonlike varicosity formed at the contact site and remained for the entire period of imaging $(8-10 \mathrm{~h})$. However filopodia continued to extend from the bouton for an additional $2-4 \mathrm{~h}$ when such motility ceased. (2) Retraction/collapse: all growth cones contacting $\mathrm{NCAM}^{-/-}$ myotubes exhibited classic growth cone collapse, as described previously (Kapfhammer and Raper, 1987; Gallarda et al., 2008). The highly active growth cones extending lamellipodia and filopodia gradually ceased such activity upon contact and the body of the growth cone collapsed, withdrawing away from the myotube. As described previously in classical growth cone collapse (Kapfhammer and Raper, 1987), in some cases one or more nonmotile filopodia maintained adhesion with the myotube and were passively stretched as the growth cone retracted. This entire process took between 15 and $60 \mathrm{~min}$. Most growth cones resumed active motility within $30-60 \mathrm{~min}$, and if one happened to touch the myotube again, this sequence of events was repeated. (3) Failure of growth cone to downregulate motility: when contacting $\mathrm{NCAM}^{-/-}$myotubes expressing the 120 isoform, growth cones did not retract. They turned and continued active growth along the myotube, but since motility was not downregulated, they grew off the ends of the myotube and failed to form a stable contact. Examples of each of these behaviors are presented below.

\section{Contact with a wild-type myotube rapidly transforms the motile motoneuron growth cone into a stable contact}

We initially assessed early motoneuron-myotube interactions as chick motoneuron growth cones approached and interacted with cultured wild-type mouse myotubes. Following initial contact (Fig. $1 A, 00.00 \mathrm{~h}$ ), the growth cone formed a bouton-like varicosity at site of initial contact (Fig. $1 B, 0.5 \mathrm{~h}$ ). Filopodia continued to extend and retract from the bouton (Fig. $1 B, 1 \mathrm{~h}$ ), but such movement stopped by $2 \mathrm{~h}$. Although the myotube moved and exhibited contractions, the bouton remained at the site of initial contact for the duration of imaging (Fig. 1A, $9.14 \mathrm{~h}$ ). This sequence occurred in six of six of the growth cone-myotube pairs that were imaged.

\section{Stable contacts do not form with NCAM-null myotubes even after $10 \mathrm{~h}$ but are rescued by exogenous expression of postsynaptic NCAM in an isoform-specific manner}

In striking contrast to the observations made on wild-type myotubes, when chick motoneuron growth cones encountered mouse myotubes that lacked all isoforms of NCAM, they failed to make stable contacts $(n=5)$, engaging in multiple cycles of making contact (Fig. 1C, 0:00 h), retracting/collapsing (1:00 $\mathrm{h})$, making contact (3:00 h), and retracting/collapsing (8:00 h). Even after $10 \mathrm{~h}$, stable contacts did not form. In this example, a nonmotile filopodium continued to adhere to the myotube as the growth cone retracted/collapsed. In other cases such filopodia were severed during the collapse. Such typical growth cone behavior upon encountering an inhibitory signal has been well described (Kapfhammer and Raper, 1987; Gallarda et al., 2008). This behavior was observed in all five growth cones that were imaged. These observations indicate that one or more isoforms of postsynaptic NCAM must be present to initiate the stable contacts described earlier.

To determine which postsynaptic NCAM isoforms were able to initiate stable contacts, individual fluorescently tagged NCAM isoforms were expressed in $\mathrm{NCAM}^{-1-}$ myotubes and the transfected myotubes were visualized by the fluorescent tags. These constructs and the resulting NCAM isoform expression have been extensively characterized previously (for details, see Materials and Methods; Hata et al., 2007). As illustrated in Figure 2A, the expression of the 140 isoform alone caused growth cones to rapidly form stable contacts in a time frame similar to that of wild-type myotubes. Two axons (arrowheads) made stable contacts by 00:10 h, and they maintained adhesion, as illustrated by the $02: 39 \mathrm{~h}$ time point, throughout the $10 \mathrm{~h}$ period of imaging, even though the myotube exhibited strong contractions/movements. These observations were made for five of six of the observed contacts. In one case, the growth cone grew over the myotube without forming a contact; however, this myotube had a relatively low level of NCAM 140 expression.

Expression of the 180 isoform was also able to rescue the formation of stable contacts. Figure $2 B$ shows a field with one 
A

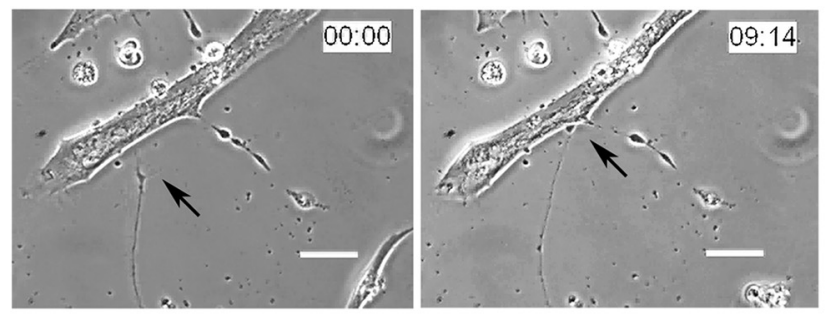

B

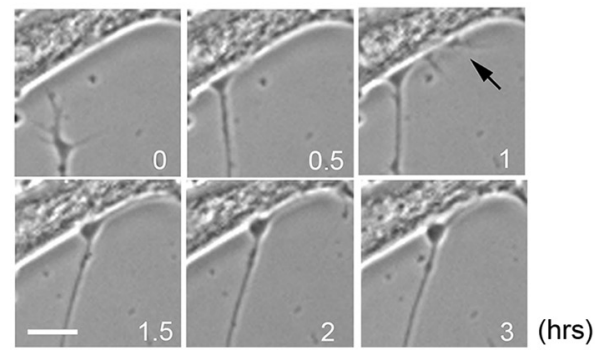

C

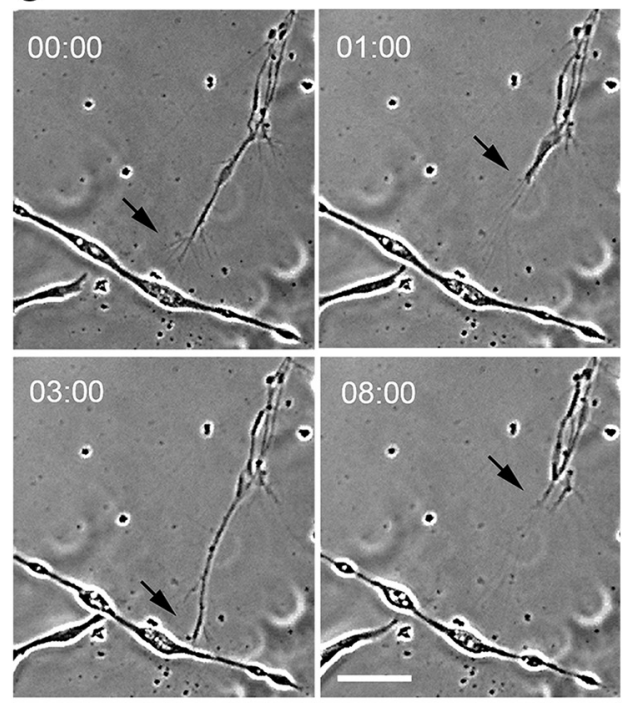

Figure 1. Motoneurons make rapid stable contacts when encountering wild-type but not NCAM-deficient mouse myotubes. Explants from the ventral spinal cord of St 25 chick embryos were cocultured with highly purified mouse wild-type myotubes for $2-4 \mathrm{~d}$ and dynamically imaged. $\boldsymbol{A}$, Two low-magnification frames from a movie at time 00 :00 $\mathrm{h}$ (defined as the moment of first filopodial contact) and after $9 \mathrm{~h}(09: 14 \mathrm{~h})$; arrows indicate growth cone and stable contact. $\boldsymbol{B}$, A series of higher-magnification images showing the motile growth cone transforming into a stable, nonmotile contact. After the formation of an enlarged presynaptic contact at $0.5 \mathrm{~h}$, filopodia of the growth cone (arrow, $1 \mathrm{~h}$ time point), remained motile exploring the area around the initial contact but by $3 \mathrm{~h}$ had ceased motility as the presynaptic contact expanded in size. No further movements were noted over the next $7 \mathrm{~h}$ of imaging. $\boldsymbol{C}$, An example of a motoneuron growth cone (arrow) from a St 25 chick ventral cord explant as it contacts an NCAM-deficient myotube (00:00 h), retracts (01:00 h), makes filopodial contact again (3:00 h), and retracts a second time (8:00 h), not forming a stable contact even after $8 \mathrm{~h}$. Scale bars: $\boldsymbol{A}, 20 \mu \mathrm{m} ; \boldsymbol{B}, 10 \mu \mathrm{m} ; \boldsymbol{C}, 20 \mu \mathrm{m}$.

NCAM 180 isoform expressing myotube (asterisk) and two NCAM-deficient myotubes (black arrowheads) with a growth cone (arrow) approaching from the bottom. By $1: 22 \mathrm{~h}$, this growth cone (arrowhead) had formed a stable contact with the NCAM $180 \mathrm{kDa}$-expressing myotube and a second growth cone had established filopodial contact (arrow) with an NCAMdeficient myotube. At 2:40 h, this second growth cone remained in contact with the NCAM-deficient myotube but had also initiated contact with the NCAM $180 \mathrm{kDa}$-expressing myotube (arrows). Although multiple filopdial contacts were made with the NCAM-deficient myotube over the next $3 \mathrm{~h}$, none established a stable contact, and by 5:40 h the growth cone had fully retracted from the NCAM-deficient myotube and had established a stable contact (arrow) with the NCAM $180 \mathrm{kDa}$-expressing myotube that remained until the end of the observation period $(8: 22 \mathrm{~h})$. Expression of the 180 isoform in an additional five myotubes rescued the formation of a stable contact.

When the GPI-linked NCAM 120 isoform was expressed in NCAM-deficient myotubes, growth cones maintained contact with the myotubes and did not retract as they did with myotubes lacking all NCAM; however, their growth cones remained highly motile (Fig. 2C, arrows), extending and retracting filopodia/lamellipodia, and usually turned to grow along the myotube for some distance before growing off the myotube. All growth cones that were imaged (seven of seven) behaved similarly. Thus, in contrast to the 180 and 140 isoforms, which have intracellular domains, the GPI-linked 120 isoform was unable to rescue stable contacts. However, its presence appeared sufficient to override an inhibitory signal that resulted in the strong growth cone retraction upon contact with NCAM-deficient myotubes. In addition to identifying NCAM-expressing myotubes by their expression of the fluorescently tagged construct, we also stained live cultures with a fluorescently conjugated NCAM antibody, NCAM 13. Use of this antibody, which recognizes all mouse NCAM isoforms but not chick isoforms, confirmed that all exogenously expressed isoforms were properly targeted to the cell surface as shown by the examples of 140 isoform-transfected myotubes (Fig. 2D; see also Hata et al., 2007).

\section{Postsynaptic expression of either the 140 or the 180 isoform of NCAM results in the formation of functional synaptic contacts}

In addition to mediating stable growth cone-myotube adhesive contacts, postsynaptic expression of either NCAM 140 or NCAM 180 isoforms resulted in the establishment of functional synaptic contacts sufficient to cause contraction. We assessed this by culturing myotubes with ventral spinal cord explants from St 25 chick embryos. These explants appeared capable of generating spontaneous bursting activity, as occurs in isolated cord preparations from either chick or mouse (Milner and Landmesser, 1999; Hanson and Landmesser, 2003, 2004). Six days after the addition of the explants to the myotube cultures, we dynamically imaged, for periods of $10 \mathrm{~min}$, fields that were close to the explant and in which axons appeared in anatomical contact with both NCAM-negative and NCAM 140, NCAM 180, or NCAM 120 $\mathrm{kDa}$-expressing myotubes.

In the example in Figure $3 A$, the lower one of the two 140 $\mathrm{kDa}$-expressing myotubes (asterisks) exhibited three spontaneous contractions during the 10 min observation period, while the other did not. None of the three NCAM-negative myotubes had spontaneous contractions. In a total of five fields, 6 of 8 of the NCAM $140 \mathrm{kDa}$-expressing myotubes exhibited contractions, ranging from 2 to 45 contractions in 10 min (mean, 13.5), while 13 of 13 NCAM-negative myotubes did not. In two cases, including that shown in Figure $3 A$, the addition of $10 \mu \mathrm{M}$ d-tubocurarine (dTC) to block neuromuscular transmission resulted in the cessation of all contractions, indicating that these were caused by functional neurotransmission. In a larger sample (Fig. 3B), we simply 

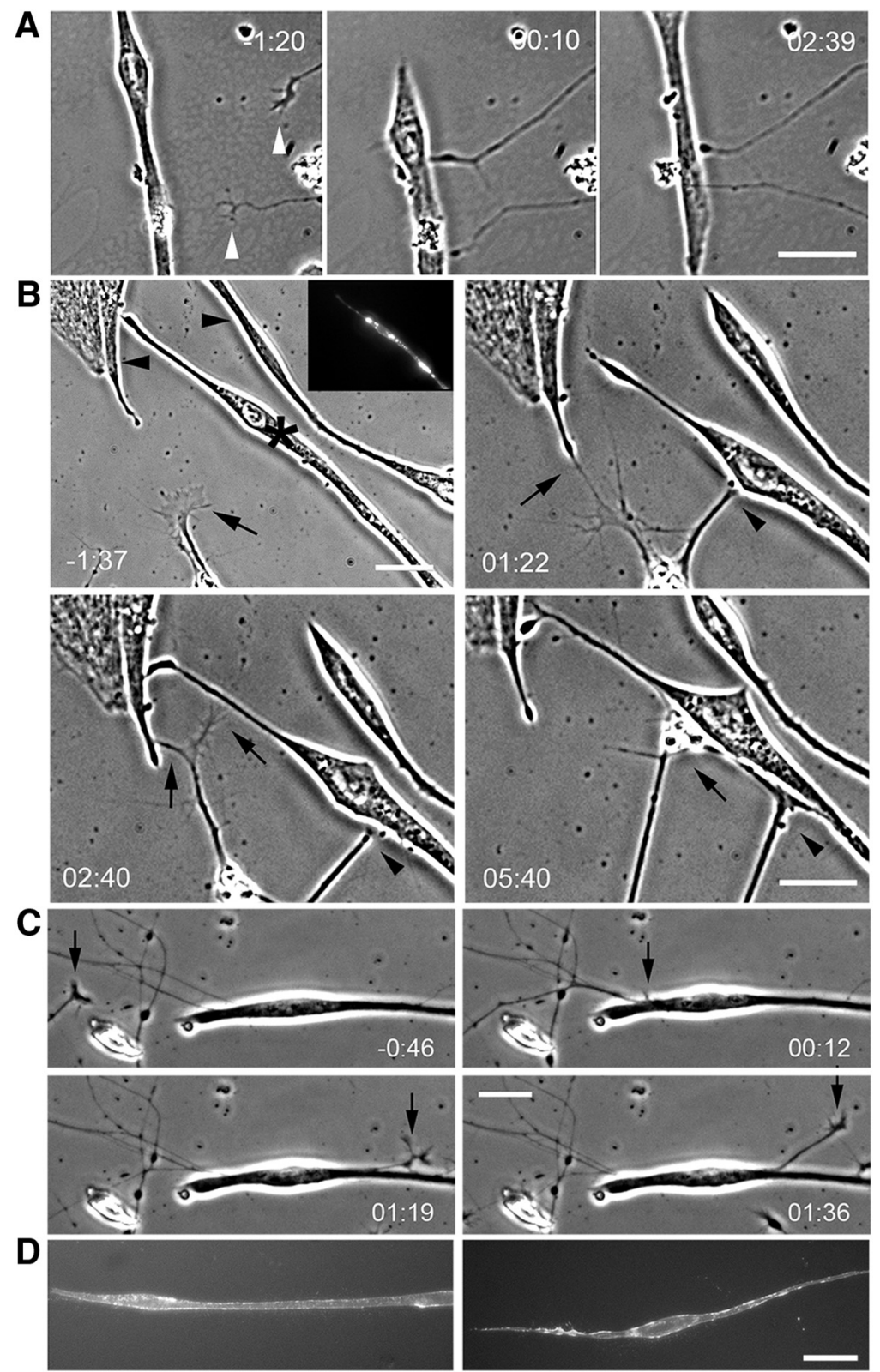

Figure 2. The ability of different postsynaptically expressed NCAM isoforms to enable stable contacts. $A$, Exogenous expression of the mouse 140 NCAM isoform in a myotube derived from NCAM ${ }^{-1-}$ mice was sufficient to rescue the formation of a stable presynaptic contact. Two growth cones (arrowheads) approached the myotube $(-01: 20 \mathrm{~h})$, made initial contact $(00: 10 \mathrm{~h})$, and maintained contact $(2: 39 \mathrm{~h})$ despite movement of the myotube. $\boldsymbol{B}$, Myotube expression of the $180 \mathrm{NCAM}$ isoform also rescued stable contacts. The first image shows a phase contrast image of a field with a 180 NCAM-expressing myotube (asterisk; see also inset showing fluorescent image of mRFP-tagged 180 NCAM) and two NCAM ${ }^{-1-}$ myotubes (arrowheads) with a growth cone (arrow) approaching from the bottom. By 1:22 h, this growth cone had made a stable contact with the NCAM 180-expressing myotube (arrowhead) and a second growth cone had made filopodial contact (arrow) with an NCAM ${ }^{-1-}$ myotube; at 2:40 $\mathrm{h}$ the second growth cone contacts both myotubes (arrows), but by 5:40 h it has retracted from the NCAM ${ }^{-1-}$ myotube, making a stable contact with the NCAM 180-expressing myotube (arrow). C, Myotube expression of the 120 NCAM isoform prevented the multiple growth cone contact-retractions seen in NCAM-negative myotubes but failed to downregulate growth cone motility. In this example, the growth cone (arrow) after making contact $(00: 12 \mathrm{~h}$ ) remained motile and turned to grow along the myotube (01: $19 \mathrm{~h}$ ), eventually growing off it $(01: 36 \mathrm{~h})$. D. Examples of two NCAM $^{-1-}$ myotubes expressing exogenous mRFP 140 NCAM, immunostained in the living state with a pan-NCAM antibody to show that the isoform is expressed at the cell surface. Scale bars, $20 \mu \mathrm{m}$.

scored whether myotubes were contracting or not. Only $5 \%$ of the NCAM-negative myotubes were contracting, and this was not significantly increased by the postsynaptic expression of the NCAM 120 isoform. However, the expression of either the NCAM 180 or 140 isoforms resulted in a statistically significant increase to $\sim 25-$
$30 \%$ of the myotubes contracting during the observation period. In summary, even after $6 \mathrm{~d}$ of coculture, NCAM-expressing motoneurons were unable to form effective functional contacts with myotubes that lacked all isoforms of NCAM. However, postsynaptic expression of the 140 or 180 isoform alone was sufficient to rescue functional synapse formation in many of the myotubes, whereas the expression of the 120 isoform was not.

As reported previously (Hata et al., 2007), when chick motoneurons make contact with chick myotubes there is a rapid accumulation of presynaptic and postsynaptic molecules involved in functional neurotransmission, including SV2, SNAP25, and AChRs. This was also observed in the present study at contacts made between chick motoneurons and wild-type mouse myotubes (data not shown). As is clear from the data presented above, very few functional contacts were formed on $\mathrm{NCAM}^{-1-}$ myotubes or on myotubes expressing only the 120 isoform. However, expression of either the 140 isoform (Fig. 3C-F, white star) or the 180 isoform (data not shown) in addition to causing stable contacts also resulted in the rapid accumulation of SV2 (Fig. 3C) and Alexa Fluor 488-conjugated $\alpha$-bungarotoxin (Fig. $3 F$ ) at the contact sites. Such accumulation did not occur on NCAMdeficient myotubes (Fig. 3, white arrowheads), even when axons (Fig. $3 C$, arrows) grew over their surfaces. Because SV2 was only weakly expressed on axons making contact with $\mathrm{NCAM}^{-/-}$myotubes, we also immunostained for SNAP25, which was more strongly expressed. In many cases, multiple axons appeared to selectively target to the NCAM-expressing myotubes (Fig. $3 D, E)$. While axons sometimes crossed over (Fig. $3 C$ ) or grew along the surface of NCAM-negative myotubes (Fig. $3 C, D$ ), multiple axons did not converge or form enlarged contacts expressing SNAP25 or SV2. To provide a more quantitative assessment of presynaptic and postsynaptic marker accumulation, the mean pixel intensity of SV2 and $\alpha$ BTX immuofluorescence was measured on NCAM 140expressing myotubes and on nearby NCAM-negative myotubes in the vicinity of axons. The mean pixel intensity of SV2 staining in arbitrary units was $6055 \pm 538$ $(n=16)$ for NCAM-deficient myotubes versus $16,476 \pm 1418(n=9)$ for NCAM 140 expressing myotubes (mean \pm SE; $p<0.01$ ). Similarly, the mean pixel intensity of $\alpha$ BTX on NCAM 140-expressing myotubes was 10,162 \pm $1683(n=7)$ versus $4592 \pm 153(n=4)$ for NCAM-negative myotubes $(p=0.037)$. We did not quantify SNAP25 levels on myotubes because, in addition to specific staining of motor ax- 
ons, nonspecific staining of myotube nuclei also occurred (Fig. 3D, asterisk), making a comparison of axonal staining on NCAM-expressing and $\mathrm{NCAM}^{-1-}$ myotubes difficult.

\section{Motoneurons that lack all NCAM isoforms turn to grow along $+/+$ myotubes but fail to stop and form stable contacts}

To assess the requirement for presynaptic NCAM in the formation of stable myotube contacts, motoneuron explants obtained from the spinal cords of E12.5 NCAM-deficient mice were cocultured with myotubes from wild-type mice and compared with cocultures in which both motoneurons and myotubes were wild type. When viewed after $6 \mathrm{~d}$, many axons in the wild-type cultures had grown out, making apparent anatomical contact with the myotubes (Fig. 4A, arrows), with some appearing to have stopped and formed stable contacts. In striking contrast, NCAMnegative axons grew, often in large numbers, up to the myotubes but then appeared to turn, avoiding contact with the myotubes and thus growing around them (Fig. 4B). This resulted in large axon fascicles running parallel to the myotube (arrows), with a relatively axon-free zone between these fascicles and the myotube. To better characterize the behavior of the motoneuron growth cones during initial myotube contact that might account for these patterns in axon outgrowth, we imaged dynamically wild-type or NCAM-deficient mouse motor axons as they contacted wildtype mouse myotubes. NCAM-containing motoneurons formed stable contacts ( 9 of 10 cases) with the wild-type myotubes, similar to the behavior of chick motor axons described earlier (Fig. 1 $A, B$ ). In contrast, while all NCAM-deficient growth cones (11 of 11) maintained contact after touching a myotube and did not retract as they did when contacting NCAM-deficient myotubes, they failed to cease motility. Usually, they turned to grow along the myotube until growing off the end (Fig. 4C,D, arrows).

To better evaluate and quantify the behavior of wild-type and NCAM-deficient motor axons when encountering wild-type myotubes, explants from E12.5 wild-type or NCAM-deficient mice were plated on cultures of wild-type mouse myotubes. After 5-6 d, they were fixed and stained with the neuron-specific tubulin antibody TuJ1 to visualize the axons and TRITC-phalloidin to stain actin filaments and thus visualize myotubes. Examples near the explant where large numbers of axons encountered the myotubes (Fig. $4 E, F$ ), show the strong convergence of the wildtype axons toward the myotube with many axons making contact (Fig. 4E). In contrast, although the NCAM-deficient axons still converged toward the myotube (Fig. $4 F$ ), many turned and grew parallel to the myotube either before the myotube or after cross- ing over it, leaving a region on either side of the myotube relatively free from axons. Such a pattern of axon fascicles parallel to the myotube would be produced by many individual growth cones failing to cease motility and turning to grow along the myotube, as shown in the examples in Figure 4, $C$ and $D$.

Although these examples provide a clear example of the different behavior of wild-type and NCAM-deficient growth cones when encountering a wild-type myotube, the density of axons precluded the quantification of the behavior of individual axons. Therefore, these were scored in myotubes further from the explant that were contacted by either a single axon or several axons, so that the behavior of individuals axons could be easily scored. The bar graph (Fig. 4G) shows that $\sim 70 \%$ of wild-type axons stopped upon myotube contact, with many fewer either crossing over the myotube or turning to grow along it. In contrast, only 15\% of the NCAM-deficient growth cones stopped upon contacting a myotube, with the large majority 

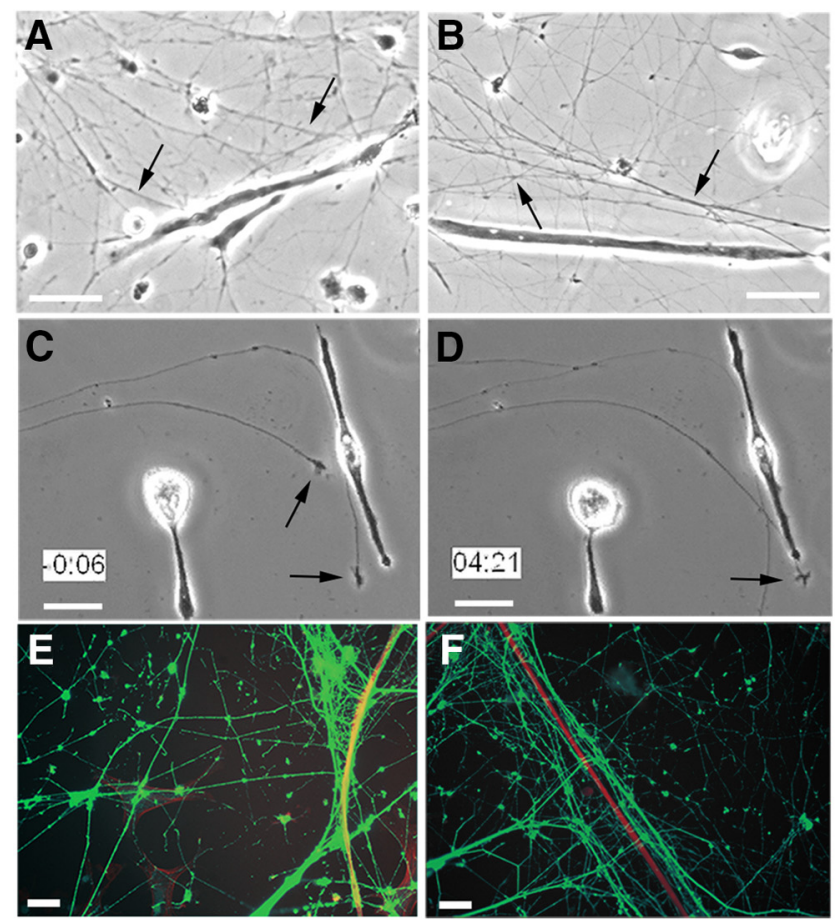

\section{G (\%)}

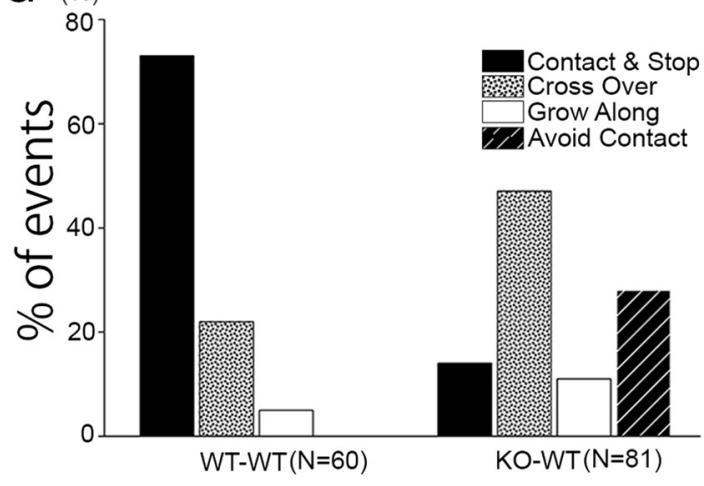

Figure 4. Mouse motoneuron growth cones lacking NCAM do not cease motility upon contact with wild-type myotubes and thus fail to make stable contacts. $A$, A phase contrast image of a $6 \mathrm{~d}$ coculture of wild-type mouse motoneurons and myotubes shows that many axons extended from explants (arrows) and came into anatomical contact with myotubes. $\boldsymbol{B}$, A similar example from a $6 \mathrm{~d}$ coculture of NCAM-deficient mouse motoneurons and wild-type mouse myotubes, showing that although there is extensive outgrowth toward the myotubes, many axons appear to have turned and grown around the myotubes, resulting in large fascicles (arrows) running parallel to the myotubes. C, D, Two frames from a movie showing NCAM-deficient growth cones (arrows) encountering a wild-type myotube. By $-00: 06 \mathrm{~h}$, one growth cone had already grown off the myotube and a second was just making contact. $D$, The latter growth cone continued to growalong the myotube, growing off by 04:21 $\mathrm{h}$ (arrow). $\boldsymbol{E}, \boldsymbol{F}$, Similar cocultures of NCAM-negative motoneurons and wild-type myotubes stained with antibodies against neuron-specific tubulin (TuJ1) to visualize axons (green) and TRITCphalloidin (red) to stain actin filaments and thus myotubes. $\boldsymbol{E}$, Wild-type motoneurons converge on and make extensive contacts with a wild-type myotube, causing the myotube to appear orange. $\boldsymbol{F}$, NCAM-deficient motoneurons also converge on a wild-type myotube, but most axons turn and grow parallel to the myotube either before or after crossing it, failing to make stable contacts. $\mathbf{G}$, Bar graph from multiple experiments quantifying the proportion of wild-type and NCAM-deficient axons that exhibit each type of behavior upon encountering a wild-type myotube (grow along: includes axons that grew in relatively close contact with myotube before or after crossing; avoid contact: includes axons that grew parallel to myotube but were not in close contact at the time of observation, but may have been earlier. Scale bars: $A, B, E, F, 40 \mu \mathrm{m} ; C, D, 20 \mu \mathrm{m}$.

either crossing over or turning and growing parallel to the myotube before or after crossing over.

We also attempted to determine whether the exogenous presynaptic expression of individual isoforms would enable axons from $\mathrm{NCAM}^{-/-}$mice to form synapses. Unfortunately, unlike St 25 chick motoneurons, we found it difficult to obtain good expression levels when E12.5 mouse motoneurons were electroporated with the different NCAM constructs and when this did occur the axons were often not in close proximity with myotubes. Nevertheless, these observations support the results of the dynamic imaging of growth cones described above and indicate the importance of presynaptic NCAM in synapse formation at the developing NMJ. Additional experiments will be required to determine whether there are any differences in the degree to which the different isoforms, expressed presynaptically, can rescue synapse formation.

Wild-type motoneuron growth cones make stable contacts on Lrp4-expressing myotubes but not on Lrp4-deficient myotubes

Lrp4, a receptor for agrin that mediates MuSK activation, is essential for NMJ formation (Weatherbee et al., 2006; Kim et al., 2008; Zhang et al., 2008; Burden et al., 2013). It was also shown to act as a retrograde signal for presynaptic differentiation of motor neurons (Yumoto et al., 2012). However, it was not known whether Lrp4 was needed at the earliest stages for motoneuron growth cones to establish initial adhesive contacts with myotubes. Cocultures of E12.5 wild-type mouse motoneurons and myotubes derived from either Lrp4-positive or Lrp4-deficient myoblast cell lines were prepared and dynamically imaged over $8 \mathrm{~h}$, as described previously for the NCAM wild-type or NCAMdeficient cocultures. As in wild-type mouse motoneuron-myotube cultures, mouse growth cones established stable adhesive contacts with the Lrp4-positive myotubes. All growth cones observed $(n=8)$ ceased motility and remained in contact with the myotube even when the latter exhibited strong movement (Fig. $5 A, 00: 00: 00$ and 02:20:00 h), or when other cells pulled on the axon, causing it to bend (Fig. 5A, 04:06:30 h, white arrowhead). In contrast, when contacting Lrp4-deficient myotubes, growth cones were either repulsed, often multiple times (Fig. $5 B ; n=17$ ), or rapidly grew over the myotube without stopping (Fig. $5 C ; n=$ 6). Thus, myotube Lrp4 is not only required for the accumulation of the presynaptic molecules needed for functional synapse formation, but also for growth cones to cease motility and form a stable adhesive contact.

\section{Lrp4 expression and AChR clustering is decreased on NCAM-deficient myotubes}

Given that wild-type growth cones behaved similarly when contacting myotubes lacking NCAM or lacking Lrp4, we determined whether Lrp4 expression might be altered on NCAM-negative myotubes. NCAM-negative or NCAM-positive myotubes were grown on a laminin substratum for 6-7 d, and after fixation and permeabilization they were stained with an antibody that recognizes the $\mathrm{N}$ terminus of Lrp4 and with Alexa Fluor 594-conjugated $\alpha \mathrm{BTX}$ to visualize AChRs.

As shown by the examples in Figure 6A, most NCAM wild-type myotubes exhibited relatively strong Lrp4 expression over much of the myotube, with staining more intense in the central regions containing nuclei and declining on either end. The staining included both relatively diffuse staining together with brighter regions/clusters. In many cases, staining was also more intense around some of the nuclei. The regions that stained for Lrp4 were also those that expressed AChRs, as visualized with $\alpha$ BTX staining. This staining included clear puncta in addition to lower levels of diffuse staining. Although AChRs (diffuse and those located in clusters) were restricted to regions with relatively high Lrp4 expression, most Lrp4 
clusters were not coincident with AChR clusters. In $\mathrm{NCAM}^{-1-}$ myotubes, Lrp4 expression was strongly downregulated, such that most of the myotube was devoid of Lrp4 staining. The Lrp4 expression remaining on some myotubes occurred in scattered puncta, as in this example, which were generally less bright than the puncta on the NCAM-positive myotubes. $\alpha$ BTX staining was even more strongly reduced, and almost no clusters were observed. The bar graph (Fig. $6 B$ ) quantifying Lrp 4 and $\alpha$ BTX staining intensity shows a strong reduction in both Lrp4 and the total number of AChRs on $\mathrm{NCAM}^{-1-}$ myotubes.

To address whether the reduction in Lrp4 expression observed via immunostaining was transcriptionally regulated we performed qPCR on cultures of $\mathrm{NCAM}^{+/+}$or $\mathrm{NCAM}^{-1-}$ myotubes. This was done for two different Lrp4 variants (Lrp4 exons 1-12 and Lrp4 exons 1-38; Fig. 6C-E). Although there was a trend for slightly increased transcription in the $\mathrm{NCAM}^{-1-}$, there was no statistically significant difference between $\mathrm{NCAM}^{+/+}$and $\mathrm{NCAM}^{-1-}$ myotubes. Thus, the reduced expression of Lrp4 observed in - / - myotubes must be at the translational or post-translational level.

To further characterize the alteration in Lrp4 levels in NCAM-deficient myotubes, an additional set of experiments was done using an adsorbed Lrp4 antibody to decrease the low level of nonspecific background staining seen with the antibody used (see Materials and Methods). Using the same fixation and permeabilization protocol as before, which should detect both cell surface and internal Lrp4, we obtained a similar pattern of staining on wild-type myotubes and confirmed the large reduction in Lrp4 expression in the NCAM-deficient myotubes (Fig. 7A). To determine whether levels of cell surface Lrp4 were also decreased, we stained live cells with the primary antibody and found a strong reduction in staining intensity when comparing NCAM-deficient to wild-type myotubes (Fig. $7 B$ ). It was also clear that cell surface Lrp4 in wild-type myotubes represented only $\sim 7 \%$ of the total Lrp4. We then determined whether the exogenous expression of individual NCAM isoforms in NCAM-deficient myotubes could rescue the expression of Lrp4. With the protocol that detected total Lrp4 staining, none of the NCAM isoforms increased the level of total Lrp4 expression (Fig. 7C). In contrast either the 120 and 140 NCAM isoforms, and to a lesser extent the 180 isoform restored Lrp4 cell surface staining toward wild-type levels (Fig. 7D). Thus, while exogenous expression of individual NCAM isoforms did not rescue overall or internal levels of Lrp4, it did restore the cell surface expression to $+/+$ levels.

We next tried to determine whether exogenous expression of Lrp4 in NCAM ${ }^{-1-}$ myotubes could rescue the formation of stable contacts and synapses on myotubes. Unfortunately, we found that cell surface Lrp4 protein levels were not increased by transfection of Lrp4 constructs to increase message levels. The intensity of Lrp4 cell surface expression in arbitrary units was $2777 \pm 337(n=12)$ for $\mathrm{NCAM}^{-1-}$ myotubes and $3550 \pm 750(n=6)$ for the Lrp4- transfected myotubes, which was not statistically significant ( $p=$ 0.289). These results suggest that exogenous increase of Lrp4 message does not result in increased cell surface Lrp4 levels. Since this seemed the relevant variable in mediating cell-cell interactions, we did not further pursue these experiments.

\section{Discussion}

We showed here that NCAM was required both presynaptically and postsynaptically for cultured chick or mouse motoneurons to form stable adhesive contacts on mouse myotubes enabling the subsequent accumulation of molecules needed for effective transmission. While NMJs form in vivo in NCAM-deficient mice, they exhibit structural and functional deficits (Rafuse et al., 2000; Polo-Parada et al., 2001, 2004). Similarly, although NCAM is not required for the formation of functionally effective mouse NMJs following reinnervation, such synapses are unstable, with presynaptic terminals subsequently withdrawing from most fast muscle fibers (Chipman et al., 2010). Presynaptic NCAM is also necessary for sprouting motor axons to reinnervate denervated muscle fibers following partial denervation (Chipman et al., 2014).

However, the requirement for specific NCAM isoforms in these processes has not been addressed. We found that motoneurons expressing all three NCAM isoforms did not form stable adhesive contacts with NCAM-deficient myotubes but instead exhibited repeated growth cone collapse/retraction, suggesting that NCAM-deficient mouse primary myotubes are inhibitory to 
A
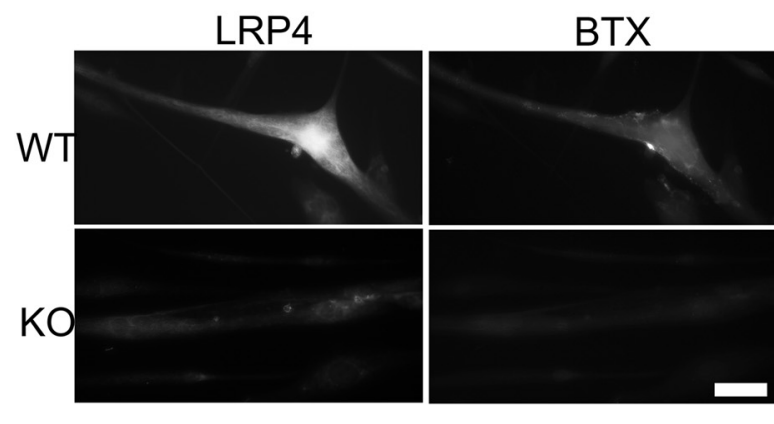

C

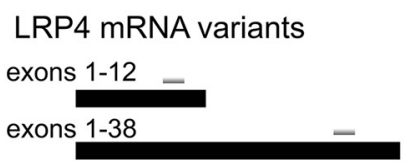

D

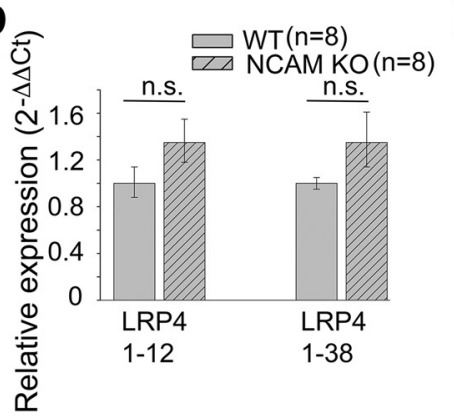

B

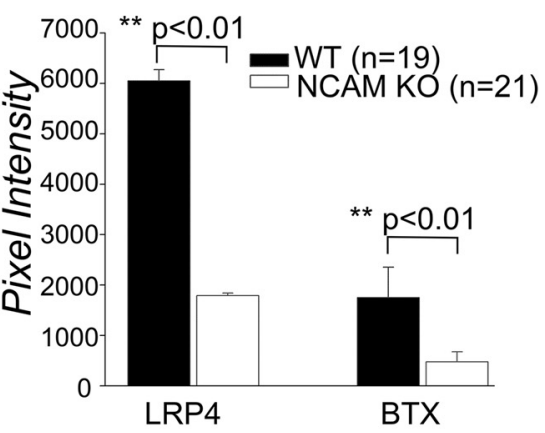

E

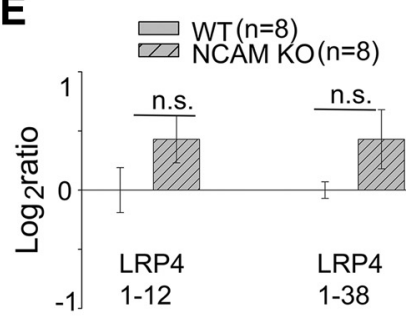

Figure 6. NCAM-deficient myotubes have reduced expression of Lrp4 and AChRs. A, Cultures of NCAM-positive and NCAM-negative myotubes, grown on laminin and costained with an antibody to Lrp4 and Alexa Fluor 488-conjugated secondary antibody and with Alexa Fluor 594-conjugated $\alpha$ BTX. Wild-type NCAM myotubes exhibited strong relatively diffuse staining for Lrp4 over much of the myotube with some puncta as well (left top). Clusters of AChRs as well as lower levels of diffuse AChR expression, visualized with $\alpha$ BTX (right top), were present on Lrp4-positive myotubes. Lrp4 expression was strongly reduced in NCAM-deficient myotubes, and its distribution was altered. Lrp4 expression was low over most of the myotube, and what expression persisted was in the form of small clusters (left bottom). $\alpha$ BTX staining on NCAM-deficient myotubes was also strongly reduced compared with wild-type myotubes, and almost no clusters were detected (right bottom). Scale bar, $10 \mu \mathrm{m}$. All panels have the same magnification. B, Bar graph quantifying the intensity of Lrp4 and $\alpha$ BTX staining for wild-type and NCAM-deficient myotubes. Myotubes were outlined manually with MetaMorph software and defined as ROIs. Total intensity of ROIs was measured and divided by the ROI area to get the average intensity over the myotube. Nineteen wild-type and 21 NCAM-deficient myotubes were measured and values are shown as the mean \pm SE. The $p$ value for LRP4 was $p=1.65 \times 10^{-8}$, and for BTX it was $p=1.08 \times 10^{-6}$. Both LRP4 and BTX staining were reduced in NCAM-deficient myotubes. C, LRP4 mRNA levels in NCAM ${ }^{+/+}$and NCAM ${ }^{-1-}$ myotubes. Two primer sets were used to detect reported variants of LRP4; exons 1-12 (Accession: NM_00145857) and LRP4 exons 1-38 (Accession: NM_ 172668). The small bar shows the location of the amplicon. $\boldsymbol{D}$, Bar graph showing the relative expression of LRP4 mRNA expression on $\mathrm{NCAM}^{+/+}$and NCAM ${ }^{-/-}$myotubes. $\boldsymbol{E}$, Bar graph showing the log 2 ratio of $L$ RP4 $\mathrm{mRNA}^{-}$expression on NCAM${ }^{+/+}$and $\mathrm{NCAM}^{-/-}$myotubes.

motoneuron growth cones. Mouse motoneurons are sensitive to various inhibitory axon guidance molecules, and muscle NCAM might modulate their response to such signals. For example, motor axons express Neuropilin-1 and their growth cones collapse in response to Sema3A, which is expressed in limb mesenchyme and differentiating muscle satellite cells (Huber et al., 2005; Tatsumi et al., 2009). Since the GPI-linked NCAM 120 isoform prevented growth cone retraction, signaling via the intracellular domains of the 140 or 180 isoform is not needed to overcome the myotube inhibitory signal. While CRMP (collapsin response mediator protein), which is required downstream of Sema3A for growth cone retraction (Schmidt and Strittmatter, 2007), binds to the intracellular domain of 180 NCAM (Büttner and Horstkorte, 2010), the modulation by NCAM of the inhibitory myotube signal must be via a different mechanism.

When chick (Dahm and Landmesser, 1991) or mouse motoneurons make initial synaptic contacts with primary myotubes in vivo, NCAM 140 is the predominant myotube isoform (Covault et al., 1986; Fredette et al., 1993), and its expression increases markedly when axons branch from the main intramuscular nerve trunks forming synapses (Landmesser et al., 1988). Here we showed that myotube expression of either the 140 or 180 isoform was sufficient to rescue the formation of stable contacts and the subsequent accumulation of presynaptic and postsynaptic molecules. In contrast, the GPI-linked 120 isoform, which becomes the predominant isoform in myotubes later in development
(Covault et al., 1986; Fredette et al., 1993), did not rescue stable contacts. While motoneuron growth cones no longer actively retracted from NCAM 120-expressing myotubes, as from NCAM-deficient ones, growth cones remained motile, growing off the myotubes. Thus, while the NCAM 120 construct used here mediated adhesion and cell aggregation in COS-7 cells (Hata et al., 2007), the signal from the myotube, triggering the cessation of growth cone motility and the formation of stable contacts, requires postsynaptic NCAM isoforms with an intracellular domain.

Our dynamic imaging revealed the following several discrete steps as motor axons encountered myotubes: (1) a rapid initial adhesion within minutes; (2) the cessation of growth cone motility within several hours; and (3) subsequent accumulation of presynaptic and postsynaptic molecules required for transmission. All three NCAM isoforms mediated initial adhesion, whereas only the 140 or 180 isoform suppressed growth cone motility enabling synapse formation. Previous studies have suggested candidate molecules that could provide the motoneuron stop signal, among these agrin, MuSK and Lrp4 (Dimitropoulo and Bixby, 2005; Kim and Burden, 2008; Yumoto et al., 2012).

Agrin is inhibitory to motor axons in culture (Bixby et al., 2002: Xu et al., 2005), and, although synapses initially formed following its genetic deletion in mice, they were unstable with axons continuing to grow along muscle fibers (Gautam et al., 1996). The more extensive overgrowth of axons and the complete 
failure of synapse formation following MuSK deletion (DeChiara et al., 1996) was more similar to our observations in cocultures when NCAM was lacking in motoneurons or the 140 and 180 isoforms were lacking in myotubes. Similar axon overgrowth occurred when the agrin receptor Lrp4 was genetically deleted in mice (Weatherbee et al., 2006; Kim et al., 2008; Zhang et al., 2008) or selectively deleted in muscle (Wu et al., 2012). Although overexpression of MuSK rescues AChR clustering in the absence of agrin, it does not rescue presynaptic differentiation in the absence of Lrp4 (Yumoto et al., 2012). Thus, Lrp4 can act as a retrograde signal for synapse formation independent of its role in agrin binding/MuSK activation. One explanation consistent with motor axons failing to stop when contacting myotubes lacking both 140 and 180 NCAM isoforms, is the downregulation of myotube Lrp4 expression. Consistent with this possibility, growth cones failed to make stable contacts when contacting myotubes lacking either Lrp4 or NCAM. Thus, in addition to its role in interacting with agrin to activate MuSK and enable the retrograde signals needed for synapse formation (Kim and Burden, 2008; Burden et al., 2013), we show that Lrp4 is also needed earlier for growth cones to stop and form stable adhesive contacts.

Lrp4 message levels detected by qPCR were not reduced on NCAM-deficient myotubes. Thus, the reduction in levels of both total and cell surface Lrp4 on NCAMdeficient myotubes must be regulated translationally or post-translationally. We also found that the expression of the NCAM 120, 140 , and, to a lesser extent, 180 isoform in NCAM-deficient myotubes restored the level of Lrp4 on the cell surface toward that of WT myotubes. However, total Lrp4 levels were not rescued by the exogenously expressed isoforms. Since cell surface Lrp4 accounts for only $\sim 7 \%$ of total Lrp4, this increase in total Lrp4 would not be detectable using immunostaining to quantify total Lrp4. These findings suggest that NCAM must affect Lrp4 levels via several different mechanisms, including as a direct or indirect chaperone for Lrp4. Nevertheless, since only cell surface Lrp4 can bind agrin or signal retrogradely, the rescue of cell surface expression could explain our observation that stable contacts and synapse formation were rescued by the expression of the 180 or the 140 NCAM isoform. However, additional signals must be involved since the 120 isoform restored cell surface Lrp4 expression but did not shut down growth cone motility.

NCAM could increase cell surface Lrp4 levels by directly or indirectly binding to Lrp4 and thus stabilizing it on the cell surface. While no evidence that NCAM binds directly to Lrp4 has been reported, it does bind MuSK (Bushell et al., 2008) and possibly other molecules that are clustered at the endplate. It is presently unclear why intracellular Lrp4 levels are greatly reduced in NCAM-deficient myotubes but are not rescued by exogenous
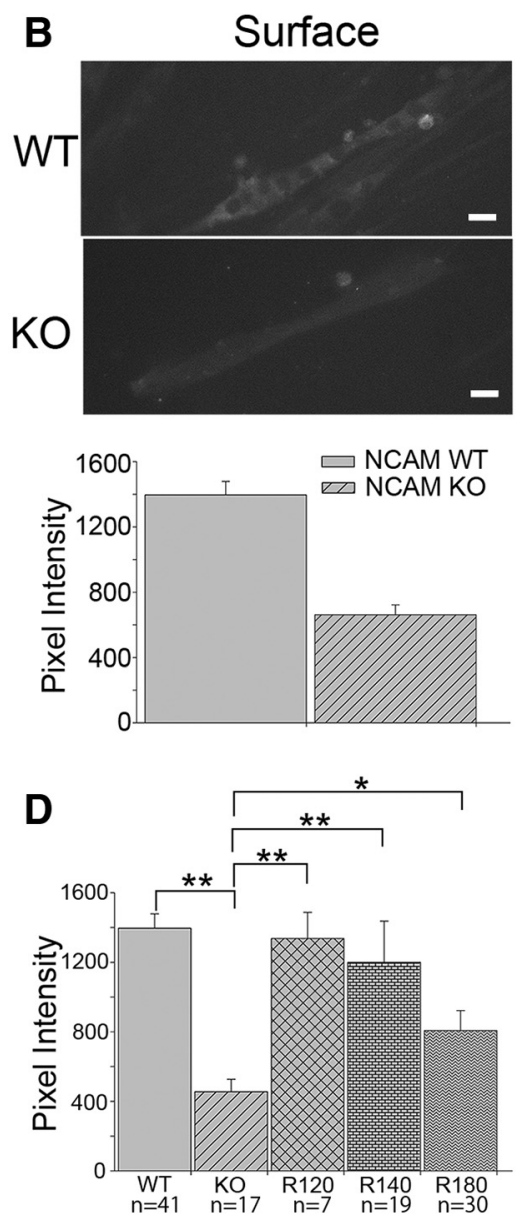

Figure 7. Reduction of total and cell surface Lrp4 levels in NCAM-deficient myotubes and rescue by exogenous expression of individual NCAM isoforms. $\boldsymbol{A}$, Immunostaining with an absorbed Lrp4 antibody (see Materials and Methods) of fixed, permeabilexpression of the 120 or the 140 NCAM isoform, and to a lesser extent the 180 isoform, restored Lrp4 cell surface levels toward WT levels. WT vs KO, $p=3.59 \times 10^{-7} ; \mathrm{KO}$ vs 120 rescue, $p=9.12 \times 10^{-6} ; \mathrm{KO}$ vs 140 rescue, $p=0.008 ; \mathrm{KO}$ vs 180 rescue, $p=0.040$. WT and $K 0$ data were reproduced from $\boldsymbol{B}$ for better comparison. Scale bars: $\boldsymbol{A}, \boldsymbol{B}, 20 \mu \mathrm{m}$.

expression of NCAM. Alterations in NCAM-deficient myotubes may occur that affect Lrp4 expression independent of NCAM or sufficient levels of exogenous NCAM may not be expressed at the time we quantified Lrp4 staining. Alternatively multiple isoforms of NCAM may be required.

Given our evidence that NCAM is required for synapse formation in motoneuron-myotube cultures, it was surprising that synapses formed in NCAM-deficient mice (Polo-Parada et al., $2001,2004)$. One explanation is that we characterized the interactions between cultured motoneurons and myotubes in isolation. In vivo, other cell types, including glia and other trophic or signaling molecules not present at appropriate levels in culture, may contribute to retrograde signaling and obviate the need for NCAM. Alternatively, in the absence NCAM, MuSK expression/ activation in cultured myotubes may be insufficient to generate the retrograde signal. In vivo, muscle prepatterning causes strong expression of MuSK in the central region of the muscle where synapses will form (Kim and Burden, 2008), and such prepatterning has not been reported in cultured myotubes. It is also 
possible that the expression of the 180 or 140 NCAM isoform enhances MuSK expression or is able to compensate in some other way for insufficient MuSK signaling.

Alternatively, the absence of NCAM throughout development, as motoneurons and muscle progenitors undergo a series of complex interactions in the in vivo environment, might trigger alterations in the expression of other molecules by the time that NMJs form in limb muscles. Previous studies, interfering with NCAM via function-blocking polyclonal antibody Fab fragments during more defined time windows, provided results similar to our current observations. In cocultures of chick myotubes and ventral spinal cord explants, anti-NCAM prevented motoneurons from making stable contacts (Rutishauser et al., 1983). Furthermore, in ovo injections of anti-NCAM Fabs into chick muscles during the formation of initial synapses (Dahm and Landmesser, 1988, 1991) greatly reduced the number and length of the collateral "synapse-forming" branches that emerge from the main intramuscular nerve trunks. It would be interesting to determine the effects of abruptly reducing NCAM expression or function in mice during a comparable period.

In summary, our observations have demonstrated the importance of different NCAM isoforms in mediating stable initial contacts between motoneuron growth cones and myotubes in culture and subsequent NMJ formation. They open up a number of interesting questions to pursue in defining underlying mechanisms, including the potential interactions between NCAM and Lrp4.

\section{References}

Bixby JL Baerwald-De la Torre K, Wang C, Rathjen FG, Rüegg MA (2002) A neuronal inhibitory domain in the $\mathrm{N}$-terminal half of agrin. J Neurobiol 50:164-179. CrossRef Medline

Burden SJ (2002) Building the vertebrate neuromuscular synapse. J Neurobiol 53:501-511. CrossRef Medline

Burden SJ, Yumoto N, Zhang W (2013) (2013) The role of MuSK in synapse formation and disease. Cold Spring Harb Perspect Biol 5:a009167. CrossRef Medline

Bushell KM, Söllner C, Schuster-Boeckler B, Bateman A, Wright GJ (2008) large-scale screening for novel low-affinity extracellular protein interactions. Genome Res 18:622-630. CrossRef Medline

Büttner B, Horstkorte R (2010) Intracellular ligands of NCAM. Adv Exp Med Biol 663:55-66. CrossRef Medline

Chipman PH, Franz CK, Nelson A, Schachner M, Rafuse VF (2010) Neural cell adhesion molecule is required for stability of reinnervated neuromuscular junctions. Eur J Neurosci 31:238-249. CrossRef Medline

Chipman PH, Schachner M, Rafuse VF (2014) Presynaptic NCAM is required for motor neurons to functionally expand their peripheral field of innervation in partially denervated muscles. J Neurosci 34:10497-110510. CrossRef Medline

Covault J, Merlie JP, Goridis C, Sanes JR (1986) Molecular forms of NCAM and its RNA in developing and denervated skeletal muscle. J Cell Biol 102:731-739. CrossRef Medline

Cremer H, Lange R, Christoph A, Plomann M, Vopper G, Roes J, Brown R, Baldwin S, Kraemer P, Scheff S (1994) Inactivation of the N-CAM gene in mice results in size reduction of the olfactory bulb and deficits in spatial learning. Nature 367:455-459. CrossRef Medline

Dahm LM, Landmesser LT (1988) The regulation of intramuscular nerve branching during normal development and following activity blockade. Dev Biol 130:621-644. CrossRef Medline

Dahm LM, Landmesser LT (1991) The regulation of synaptogenesis during normal development and following activity blockade. J Neurosci 11:238255. Medline

DeChiara TM, Bowen DC, Valenzuela DM, Simmons MV, Poueymirou WT, Thomas S, Kinetz E, Compton DL, Rojas E, Park JS, Smith C, DiStefano PS, Glass DJ, Burden SJ, Yancopoulos GD (1996) The receptor tyrosine kinase MuSK is required for neuromuscular junction in vivo. Cell 85:501512. CrossRef Medline

Dimitropoulou A, Bixby JL (2005) Motor neurite outgrowth is selectively inhibited by cell surface MuSK and agrin. Mol Cell Neurosci 28:292-302. CrossRef Medline
Fox MA, Ho MS, Smyth N, Sanes JR (2008) A synaptic nidogen: developmental regulation and role of nidogen-2 at the neuromuscular junction. Neural Dev 3:24. CrossRef Medline

Fredette B, Rutishauser U, Landmesser L (1993) Regulation and activitydependence of NCAM isoforms and polysialic acid on chick myotubes during development. J Cell Biol 123:1867-1888. CrossRef Medline

Gallarda BW, Bonanomi D, Müller D, Brown A, Alaynick WA, Andrews SE, Lemke G, Pfaff SL, Marquardt T (2008) Segregation of axial motor and sensory pathways via heterotypic trans-axonal signaling. Science 320: 233-236. CrossRef Medline

Gautam M, Noakes PG, Moscoso L, Rupp F, Scheller RH, Merlie JP, Sanes JR (1996) Defective neuromuscular synaptogenesis in agrin-deficient mutant mice. Cell 85:525-535. CrossRef Medline

Hagiwara Y, Ozawa E (1994) A new method for fibroblast-less primary skeletal muscle cell culture by the use of hydroxyurea. Dev Growth Differ 36:141-148. CrossRef

Hanson MG, Landmesser LT (2003) Characterization of the circuits that generate spontaneous episodes of activity in the early embryonic mouse spinal cord. J Neurosci 23:587-600. Medline

Hanson MG, Landmesser LT (2004) Normal patterns of spontaneous activity are required for correct motor axon guidance and the expression of specific guidance molecules. Neuron 43:687-701. CrossRef Medline

Hata K, Polo-Parada L, Landmesser LT (2007) Selective targeting of different neural cell adhesion molecule isoforms during motoneuron myotube synapse formation in culture and the switch from an immature to mature form of synaptic vesicle cycling. J Neurosci 27:14481-14493. CrossRef Medline

Huber AB, Kania A, Tran TS, Gu C, De Marco Garcia N, Lieberam I, Johnson D, Jessell TM, Ginty DD, Kolodkin AL (2005) Distinct roles for secreted semaphorin signaling in spinal motor axon guidance. Neuron 48:949964/ CrossRef Medline

Kapfhammer JP, Raper JA (1987) Collapse of growth cone structure on contact with specific neurites in culture. J Neurosci 7:201-212. Medline

Kim N, Burden SJ (2008) MuSK controls where motor axons grow and form synapses. Nat Neurosci 11:19-27. CrossRef Medline

Kim N, Stiegler AL, Cameron TO, Hallock PT, Gomez AM, Huang JH, Hubbard SR, Dustin ML, Burden SJ (2008) Lrp4 is a receptor for Agrin and forms a complex with MuSK. Cell 135:334-342. CrossRef Medline

Landmesser L, Dahm L, Schultz K, Rutishauser U (1988) Distinct roles for adhesion molecules during innervation of embryonic chick muscle. Dev Biol 130:645-670. CrossRef Medline

Lin W, Burgess RW, Dominguez B, Pfaff SL, Sanes JR, Lee KF (2001) Distinct roles of nerve and muscle in postsynaptic differentiation of the neuromuscular synapse. Nature 410:1057-1064. CrossRef Medline

Maness PF, Schachner M (2007) Neural recognition molecules of the immunoglobulin superfamily: signaling transducers of axon guidance and neuronal migration. Nat Neurosci 10:19-26. CrossRef Medline

Milner LD, Landmesser LT (1999) Cholinergic and GABAergic inputs drive patterned spontaneous motoneuron activity before target contact. J Neurosci 19:3007-3022. Medline

Nishimune H, Valdez G, Jarad G, Moulson CL, Müller U, Miner JH, Sanes JR (2008) Laminins promote postsynaptic maturation by an autocrine mechanism at the neuromuscular junction. J Cell Biol 182:1201-1215. CrossRef Medline

Polo-Parada L, Bose CM, Landmesser LT (2001) Alterations in transmission, vesicle dynamics, and transmitter release machinery at NCAM-deficient neuromuscular junctions. Neuron 32:815-828. CrossRef Medline

Polo-Parada L, Bose CM, Plattner F, Landmesser LT (2004) Distinct roles of different neural cell adhesion molecule (NCAM) isoforms in synaptic maturation revealed by analysis of NCAM $180 \mathrm{kDa}$ isoform-deficient mice. J Neurosci 24:1852-1864. CrossRef Medline

Rafuse VF, Polo-Parada L, Landmesser LT (2000) Structural and functional alterations of neuromuscular junctions in NCAM-deficient mice. J Neurosci 20:6529-6539. Medline

Rando TA, Blau HM (1994) Primary mouse myoblast purification, characterization, and transplantation for cell-mediated gene therapy. J Cell Biol 125:1275-1287. CrossRef Medline

Rutishauser U, Grumet M, Edelman GM (1983) Neural cel adhesion molecule mediates initial interactions between spinal cord neurons and muscle cells in culture. J Cell Biol 97:145-152. CrossRef Medline

Sanes JR, Lichtman JW (2001) Induction, assembly, maturation and 
maintenance of a postsynaptic apparatus. Nat Rev Neurosci 2:791805. CrossRef Medline

Schmidt EF, Strittmatter SM (2007) The CRMP family of proteins and their role in Sema3A signaling. Adv Exp Med Biol 600:1-11. CrossRef Medline

Shetty A, Sytnyk V, Leshchyns'ka I, Puchkov D, Haucke V, Schachner M (2013) The neural cell adhesion molecule promotes maturation of the presynaptic endocytic machinery by switching synaptic vesicle recycling from adaptor protein 3 (AP-3)- to AP-2-dependent mechanisms. J Neurosci 33:16828-16845. CrossRef Medline

Stork O, Welzl H, Cremer H, Schachner M (1997) Increased intermale aggression and neuroendocrine reponse in mice deficient for the neural cell adhesion molecule (NCAM). Eur J Neurosci 9:1117-1125. CrossRef Medline

Tatsumi R, Sankoda Y, Anderson JE, Sato Y, Mizunoya W, Shimizu N, Suzuki T, Yamada M, Rhoads RP Jr, Ikeuchi Y, Allen RE (2009) Possible implication of satellite cells in regenerative motoneuritogenesis: HGF upregulates neural chemorepellent Sema3A during myogenic differentiation. Am J Physiol Cell Physiol 297:C238-C252. CrossRef Medline
Weatherbee SD, Anderson KV, Niswander LA (2006) LDL-receptor related protein 4 is crucial for formation of the neuromuscular junction. Development 133:4993-5000. CrossRef Medline

Wu H, Xiong WC, Mei L (2010) To build a synapse:signaling pathways in neuromuscular junction assembly. Development 137:1017-1033. CrossRef Medline

Wu H, Lu Y, Shen C, Patel N, Gan L, Xiong WC, Mei L (2012) Distinct roles of muscle and motoneuron Lrp4 in neuromuscular junction formation. Neuron Neuron 75:94-107. CrossRef Medline

Xu X, Fu AK, Ip FC, Wu CP, Duan S, Poo MM, Yuan XB, Ip NY (2005) Agrin regulates growth cone turning of Xenopus spinal motoneurons. Development 132:4309-4316. CrossRef Medline

Yumoto N, Kim N, Burden SJ (2012) Lrp4 is a retrograde signal for presynaptic differentiation at neuromuscular synapses. Nature 489:438-442. CrossRef Medline

Zhang B, Luo S, Wang Q, Suzuki T, Xiong WC, Mei L (2008) Lrp4 serves as a coreceptor of agrin. Neuron 60:285-297. CrossRef Medline 